exposures and outcomes of interest from prior literature to be examined at a level of detail not possible in the original study, e.g. nerve agent antidotes, riot control agents, neurological mortality, and rarer cancers.

Implications Greater understanding of the long-term risks associated with exposure to chemical agents could lead to raised awareness among policy makers and health care providers, leading to improved diagnosis and quality of care.

Conflict of interest None

\section{P.2.03 DESIGN, DEVELOPMENT AND IMPLEMENTATION OF A NATIONAL MULTI-SITE FIRE FIGHTER CANCER COHORT STUDY}

\begin{abstract}
${ }^{1}$ Alberto Caban-Martinez, ${ }^{2}$ Kenny Fent, ${ }^{3}$ Casey Grant, ${ }^{1}$ Natasha Schaefer Solle, ${ }^{4}$ Elizabeth Jacobs, ${ }^{5}$ Sara Jahnke, ${ }^{4}$ Stephanie Griffin, ${ }^{6}$ Gavin Horn, ${ }^{3}$ Rita Fahy, ${ }^{7}$ Patrick Morrison, ${ }^{2}$ Steve Bertke, ${ }^{4}$ Kyoung Sook Jeong, ${ }^{4}$ Jin Zhou, ${ }^{4}$ Sally Littau, ${ }^{4}$ Alesia Jung, ${ }^{4}$ Jeffrey Burgess. ${ }^{1}$ University of Miami, Miller School of Medicine, Miami, USA; ${ }^{2}$ National Institute for Occupational Safety and Health, Cincinnati, USA; ${ }^{3}$ National Fire Protection Association, Quincy, USA; ${ }^{4}$ University of Arizona, Mel and Enid Zuckerman College of Public Health, Tucson, USA; ${ }^{5}$ National Development and Research Institutes, Inc., New York, USA; ${ }^{6}$ Illinois Fire Service Institute, Champaign, USA; ${ }^{7}$ International Association of Fire Fighters, Washington, USA
\end{abstract}

\subsection{6/OEM-2019-EPI.239}

Background and objective(s) Retrospective epidemiologic studies suggest a disproportionate burden in specific cancer incidence rates in U.S. fire fighters when compared to the general population. Exposure to hazardous chemicals and carcinogenic compounds during fire incident response may be contributing to these observed elevated cancer rates. Research studies that prospectively collect and integrate exposure, biomarker and health survey information within the fire fighter workforce are lacking. In the present study, we 1) describe the design and development of a multi-state prospective fire fighter cancer cohort study; and 2) discuss the collection of cancer biomarker data from the first fire department.

Methods In July 2016, through FEMA-funding a national multi-state prospective study was designed with biomarker, exposure and data collection cores. Each core is comprised of a team of multidisciplinary investigators across governmental, industry and academic institutions with instrumentation and resources to collect field measurements across fire service departments. An oversight and planning board was empaneled with fire fighter department and union leadership and subject matter experts to guide the design, collection and analysis of integrated data streams.

Results A total of 62 new recruits from a large U.S. large career Fire Department were consented and enrolled into the Fire Fighter Cancer Cohort Study in February 2018. Among consenting rookie firefighters 60 consented (response rate 60/ $62=96.8 \%)$ to the optional biomarker and exposure collection protocols as well as granted permission for follow up in the future. Two phlebotomists and an occupational health nurse at the training academy collected a total of 5 tubes from each firefighter (i.e., TEMPUS, PAXGENE RNA, Sterile Red Top, EDTA, and sodium citrate tube).

Conclusions The newly established national prospective cancer cohort study infrastructure supports the collection of electronic consent, biomarker, exposure and health survey data. Expansion of the research protocol to other firefighter subgroups in needed.

\section{P.2.04 OCCUPATIONAL SKIN DISEASE IN A TERTIARY OCCUPATIONAL DERMATOLOGIC CLINIC: A RETROSPECTIVE ANALYSIS FROM 2010 TO 2017}

Heng-Hao Chang* ${ }^{*}$ Bour-Jr Wang, How-Ran Guo, I-Ru Lee. National Cheng Kung University Hospital, Tainan, Taiwan

\subsection{6/OEM-2019-EPI.240}

Background Occupational skin disease (OSD) is one of the most common occupational disorders in Taiwan. As reporting OSD was not compulsory, there was limited information on the exact causes and patient characteristics. The objective of this study was to investigate the causes and common allergens among OSD patients in Taiwan.

Methods We recruited patients from Occupational Dermatology Clinic in National Cheng Kung University Hospital, a tertiary referral center in Tainan city, between 1 January 2010 and 31 July 2017. Patch testing with European baseline series, additional occupation-oriented series, and personal material exposed at work or during daily life was carried out if the patients were suspected of allergic skin diseases.

Results Among the 273 patients who received patch testing, $51(18.7 \%)$ patients had a final diagnosis of OSD. $82.4 \%$ of the 51 patients were diagnosed with allergic contact dermatitis (ACD), 11.8\% with irritant contact dermatitis (ICD), while the rest $5.9 \%$ with both. Patient reported 3.0 years of skin problem prior to the clinic visit. The vast majority of patients suffered from hand eczema. Epoxy resin workers, food workers, hairdressers and beauty salon beauticians were the most common occupations related to OSD. The most important allergens were nickel, fragrance mix I, potassium dichromate and paraben mix. Around half of the patients showed allergic reaction to their personal material.

Discussion As worker compensation statistics may not accurately estimate the characteristics of OSD patients, our study was crucial to identify the high-risk groups as well as the common allergens related to their work. Although the results might not represent the proportion of patients of OSD in other clinic, drawing information from patch testing may reflect those patients of severer or longer duration of symptoms. Future occupational measures should be taken on these industries for the detection and prevention of OSD.

\section{P.2.06 RISK AND PREVENTION OF SENSITIZATION AND ALLERGIC CONTACT DERMATITIS AMONG WORKERS HANDLING EPOXY RESINS}

${ }^{1}$ Alexandra Golabek Christiansen* ${ }^{*}{ }^{2}$ Ole Carstensen, ${ }^{3}$ Mette Sommerlund, ${ }^{4} \mathrm{Per}$ Axel Clausen, ${ }^{4,5}$ Vivi Schlünssen, ${ }^{6} J a k o b$ Bønløkke, ${ }^{1}$ Charlotte Amalie Ihlo, ${ }^{7}$ Marléne Isaksson, ${ }^{8}$ Pia Christoffersen, ${ }^{9}$ Christian Libak, ${ }^{9}$ Marcus Illanes, ${ }^{1}$ Henrik Kolstad. ${ }^{1}$ Department of Occupational Medicine, Aarhus University Hospital, Aarhus, Denmark; ${ }^{2}$ Department of Occupational Medicine, The Regional Hospital West Jutland, Herning, Denmark; ${ }^{3}$ Department of Dermatology, Aarhus University Hospital, Aarhus, Denmark; ${ }^{4}$ National Research Center for the Working Environment, Copenhagen, Denmark; ${ }^{5}$ Department of Public Health, Aarhus University, Aarhus, Denmark; ${ }^{6}$ Department of Occupational and Environmental Medicine, Aalborg University Hospital, Aalborg, Denmark; ${ }^{7}$ Department of Occupational and Environmental Dermatology, Skåne University Hospital, Malmö, Sweden; ${ }^{8}$ Vestas Wind Systems A/S, Lem, Denmark; ${ }^{9}$ Siemens Gamesa Renewable Energy, Aalborg, Denmark

10.1136/OEM-2019-EPI.241

High prevalences of sensitization and allergic contact dermatitis (ACD) have been reported among workers exposed to 